\title{
Effects of a Cognitive Distraction Task on Gait Mechanics in Recently Gait-Retrained Runners
}

\author{
Erin E. Futrell, PT, MPT, OCS ${ }^{*}$ (D) Darcy S. Reisman, PT, PhD', David R Mullineaux, $P h D^{3}$ and Irene $S$. \\ Davis, PT, PhD ${ }^{4}$
}

${ }^{1}$ Department of Physical Therapy, Springfield College, USA

${ }^{2}$ Department of Physical Therapy, University of Delaware, USA

${ }^{3}$ School of Sport and Exercise Science, University of Lincoln, UK

${ }^{4}$ Department of Physical Medicine and Rehabilitation, Harvard Medical School, USA

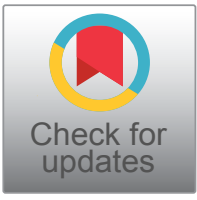

*Corresponding author: Erin E. Futrell, PT, MPT, OCS, Department of Physical Therapy, Springfield College, 263 Alden Street, Springfield, MA 01109, USA

\begin{abstract}
Background: When assessing running mechanics in a laboratory setting, there is risk for performance bias by the participants. The purpose of this study was to determine if runners who had recently undergone gait retraining altered their running mechanics while performing a cognitive distraction task.

Methods: Two groups of runners were analyzed at 3 postintervention time-points for changes in vertical loadrates, cadence, and footstrike pattern.

Results: Runners who received cadence retraining (CAD) did not alter cadence, but had increased loadrates when distracted. Runners who received footstrike retraining (FFS) did not alter foot angle or loadrates while distracted. However, both increases and decreases were found in cadence and foot angle in response to distraction.
\end{abstract}

Conclusions: Utilizing a distraction task with gait analyses, especially post-gait retraining, is feasible and recommended.

\section{Keywords}

Running, Dual-task, Physical therapy, Biomechanics

\section{Introduction}

The majority of running gait analyses are conducted in a laboratory setting. However, there is a question of the ecological validity in this type of research due to the runner's awareness of being observed and analyzed. Ideally, runners would be measured in their natural running environments by using wearable sensors. However, until there is greater evidence of the reliability and validity of such technology, the laboratory setting remains the gold standard for assessing running gait mechanics [1].

Bias and threats to validity that may occur during research include study design, data collection, and data analysis and interpretation. Random allocation and blinding researchers and participants to treatments are ways to limit bias and validity threats. Biomechanists use calibrated equipment to objectively measure gait characteristics, but bias associated with human behavior during data collection is more difficult to control.

Bias can be introduced into a study when people know they are being monitored. This phenomenon is known as the Hawthorne Effect, and has been referred to more recently as participant reactivity [2,3]. This type of performance bias can occur because participants may alter their behavior based on the knowledge they are being observed. This has been reported in a small observational gait study of individuals with prosthetic devices. Specifically, these authors found several changes in gait patterns when participants were aware of the presence of observers [4].

Participant reactivity can confound the measurement of an intervention effect as well. Gait retraining of faulty

Citation: Futrell EE, Reisman DS, Mullineaux DR, Davis IS (2022) Effects of a Cognitive Distraction Task on Gait Mechanics in Recently Gait-Retrained Runners. Int J Sports Exerc Med 8:215. doi. org/10.23937/2469-5718/1510215

Accepted: February 24, 2022; Published: February 26, 2022

Copyright: (C) 2022 Futrell EE, et al. This is an open-access article distributed under the terms of the Creative Commons Attribution License, which permits unrestricted use, distribution, and reproduction in any medium, provided the original author and source are credited. 
running mechanics is a frequent intervention used by physical therapists. Its purpose is to reduce the risk of a running-related injury, and therefore it is important to determine if motor learning has occurred, and if it persists over time. As it is difficult to blind participants when asking them to alter gait mechanics, the probability of introducing experimental error in gait mechanics data is high. Even with observational running analyses, in which no intervention is administered, there may be some level of participant reactivity due to the unnatural laboratory environment in which runners are asked to perform. However, introducing a distraction task while assessing one's mechanics may reduce the ability for the participant to consciously perform for the tester. Additionally, runners may have to attend to an array of visual and auditory distractions when running outdoors. Therefore, use of a distraction task in a laboratory setting may provide enhanced ecologically valid data. Studies of jump-landing performance and obstacle-avoidance during walking revealed significant changes in lower extremity mechanics when participants performed a secondary cognitive task $[5,6]$. However, the effect of distraction on either habitual or retrained running mechanics has not been examined.

Therefore, the purpose of the current study was to determine if running mechanics that had recently been retrained would be altered under a distracted condition. We also sought to determine whether there was a learning effect of the distraction task itself when administered over multiple time points. We hypothesized that newly retrained gait mechanics within two intervention groups would alter under a distracted condition. Specifically, that the group trained to increase cadence (number of steps/minute) would reduce cadence toward pre-gait retraining levels, and the group who received transition to forefoot strike training would reduce plantar flexion foot angle at contact with distraction. We hypothesized that because of these gait alterations, both groups would have increased vertical load rates with distraction. We also expected that the distraction task would be equally disruptive over multiple time points due to the brevity of the task ( 20 seconds) and the length of time between each gait assessment.

\section{Methods}

The institutional review board approved this investigation and participants gave consent before any research activity occurred. This study is part of a larger one comparing the short and long-term effects of two types of gait retraining. These were increasing cadence and transitioning from a Rearfoot Strike (RFS) to a forefoot strike pattern. Both of these methods have been shown to reduce vertical loadrates, which have been related to a variety of running related injuries [7-9]. Thirty-three healthy recreational runners volunteered for this study. In brief, runners were randomized into
Table 1: Participant demographics.

\begin{tabular}{|l|l|l|}
\hline FFS (n=15) & CAD (n =18) \\
\hline Age (yrs) & $30 \pm 5.5$ & $30 \pm 6.3$ \\
\hline Height (m) & $1.68 \pm 0.06$ & $1.65 \pm 0.09$ \\
\hline Weight (kg) & $71.68 \pm 11.35$ & $64.01 \pm 11.73$ \\
\hline Distance (km/week) & $14.64 \pm 4.94$ & $15.07 \pm 4.34$ \\
\hline Self-selected speed (m/s) & $2.51 \pm 0.19$ & $2.61 \pm 0.15$ \\
\hline Sex & $5 \mathrm{M}, 10 \mathrm{~F}$ & $4 \mathrm{M}, 14 \mathrm{~F}$ \\
\hline
\end{tabular}

an increased cadence (CAD) or transition to forefoot strike (FFS) group. To be included, participants had to be running distances of $8-24 \mathrm{~km} /$ week for the past 3 months and with no injury for the past 3 months. They were further screened and those who ran with a rearfoot strike pattern with a cadence of $\leq 170 \mathrm{spm}$ (steps/min) were invited to participate (Table 1 ).

Participants underwent a baseline gait analysis followed by instruction in a 4-week foot and ankle strengthening program. Both groups received 8 gaitretraining sessions over a 2-3 week period using auditory feedback. As a result, the CAD group increased their cadence and the FFS group transitioned to a FFS pattern with a plantarflexed foot angle at initial contact [10]. Following gait retraining, runners were provided written instructions on how to gradually increase their distance. Post-intervention gait analyses were then performed 1 week post-retraining and at 1 and 6 month post-retraining.

For the purpose of this investigation, only the postretraining gait assessments were analyzed to assess the effect of distraction on the retrained gait patterns. All gait analyses were performed on an instrumented treadmill (AMTI, Watertown, MA). Retroreflective markers were placed on the calcaneus through holes in the laboratory-issued neutral cushioned shoes (Nike Air Pegasus) to assess foot angle with respect to the ground. Kinematic data were recorded using an 8-camera motion capture system (Vicon, Oxford, United Kingdom) sampled at $250 \mathrm{~Hz}$, and kinetic data were recorded using the instrumented treadmill sampled at $1500 \mathrm{~Hz}$. After a 3-minute warm- up, speed was adjusted to each participant's self-selected long run pace, and 20 seconds of data were recorded.

To assess the effects of participant reactivity, participants continued running at the same pace, and were recorded a second time for 20 seconds while performing a cognitive distraction task. This task was a version of the Stroop Test, and was intended to distract runners from focusing on their gait while being recorded [11]. While running, a television monitor directly in front of the treadmill displayed a random 7 row $\times 5$ column written list of names of colors, in which the color of the text did not match the name of the color. Runners were instructed to read down each column and say aloud the color of the text, but not the word itself. For example, if 


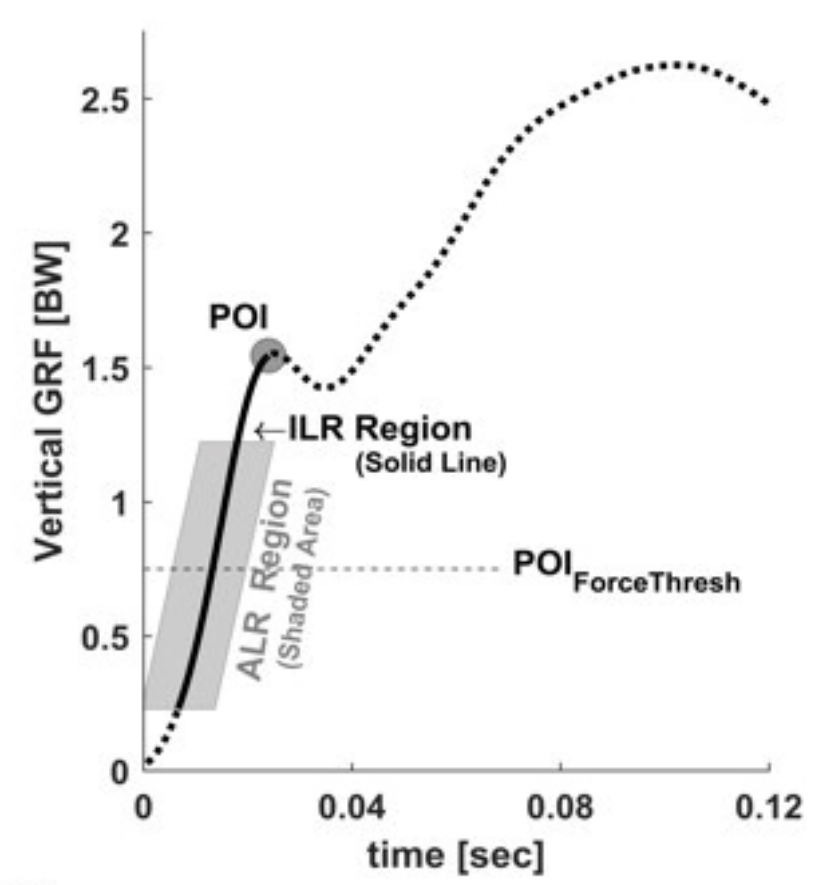

A)

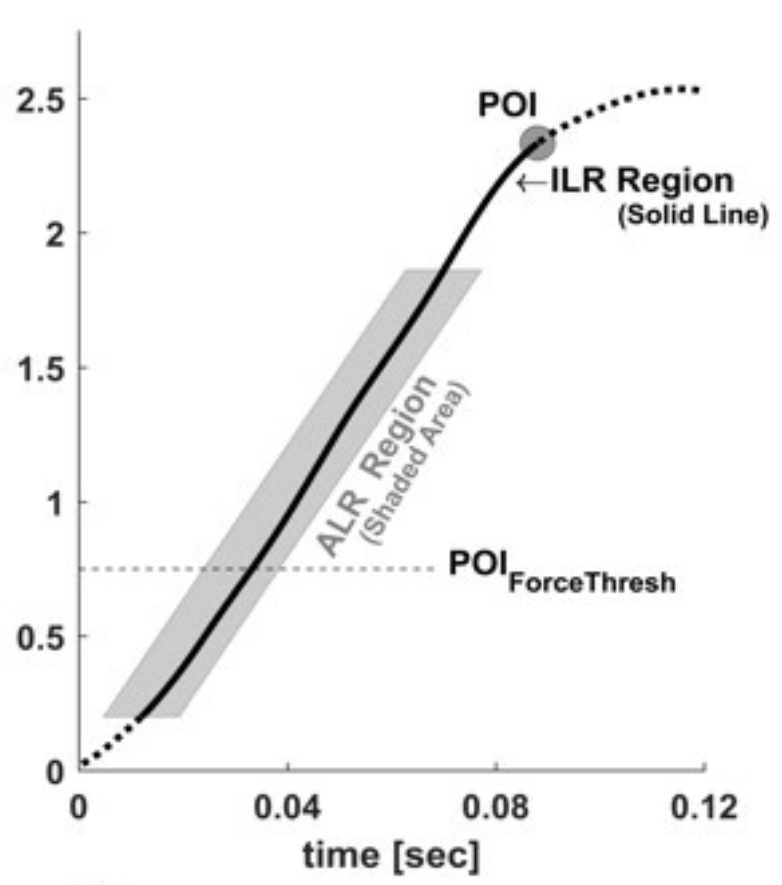

B)

Figure 1: VGRF curve with average and instantaneous vertical loadrates (ALR and ILR) calculations using point of interest (POI). A) Curve with impact peak; B) Curve with no impact peak (not pictured: curve with impact transient).

the word "black" was written in green text, the correct response was "green". Participants first practiced the distraction task while standing stationary on the treadmill to ensure they understood the requirements. This version of the Stroop Test was not timed, nor scored for accuracy. Runners were encouraged to simply read the chart at their own pace. This was done to reduce the mental stress and cognitive demand of the task.

The mean values for the first 10 right foot strikes in each trial were used for data analysis. All data were lowpass filtered using a fourth-order Butterworth filter with a frequency cutoff of $12 \mathrm{~Hz}$ for kinematic data and 50 $\mathrm{Hz}$ for kinetic data. Data were processed using Visual 3D software (C-motion, Germantown, MD) and customized MATLAB code (MathWorks, Natick, MA).

Variables of interest were cadence for the CAD group, foot angle at initial contact for the FFS group, and vertical average and instantaneous loadrates (VALR, VILR) for both groups. To determine loadrates using the Vertical Ground Reaction Force (VGRF) curve, three types of curves were identified: One with a vertical impact peak, one with an impact transient with a more level slope, and one with neither a peak nor transient. For each type of curve, a Point of Interest (POI) was used to define a range over which the vertical loadrates would be calculated. The POI was the first point greater than $75 \%$ of a participant's body weight, and less than $15 \mathrm{BW} / \mathrm{s}$. This criterion was used to justify an impact transient or end of loading (Figure 1). The VALR region was defined as the average slope between $20-80 \%$ of the curve between footstrike and POI. The VILR was defined as the peak slope between any two successive points between $20-100 \%$ of the curve from footstrike to POI. Foot angle was determined by the angle between the lateral foot and the laboratory coordinate system, with zero degrees defined as the angle achieved during a static standing trial. During running, a negative angle indicated a FFS (plantarflexion), and a positive angle indicated a RFS (dorsiflexion) at initial contact. Cadence was calculated as the number of steps per minute, and was extracted from the kinetic data.

Statistical analysis was performed using SPSS Version 25 , with level of significance for all variables set as 0.05 . The presence of outliers for each variable was assessed. If present, outliers were removed using the "Median Absolute Deviation" (MAD) method [12]. Modifications to the MAD were applied for more stringent criteria of scaling MAD using a maximum t-statistic of 0.001 [13]. The number of participants included for each variable's analysis is listed in Table 2.

We used a 2 condition (undistracted vs. distracted) $\times 3$ time (post-intervention time points) ANOVA in order to address the aims of this study. The condition $x$ time interaction provided results regarding whether learning of the distraction task had occurred over time for each variable of interest (cadence, foot angle, and vertical loadrates). If not, the main effects of condition were assessed to determine whether these variables of interest changed under distraction. Mauchly's test of sphericity indicated the assumption of sphericity was met for interaction effects for all variables of interest.

In order to further explore the data, we examined the individual participant data. Specifically, we calculated 
Table 2: Results of $2 \times 3$ ANOVA for within group changes between undistracted and distracted running across three post gaitretraining analyses.

\begin{tabular}{|c|c|c|c|c|c|c|}
\hline CAD GROUP & Time point & Undistracted & Distracted & $\begin{array}{l}\text { Effect size } \\
\text { (Cohen's d) }\end{array}$ & Condition effect & $\begin{array}{l}\text { Time-condition } \\
\text { effect }\end{array}$ \\
\hline \multirow{3}{*}{$\begin{array}{l}\text { Cadence (steps/min) } \\
\mathrm{n}=16\end{array}$} & $1 \mathrm{wk}$ & $173.4(6.9)$ & $171.7(7.9)$ & 0.2 & \multirow{3}{*}{0.493} & \multirow{3}{*}{0.09} \\
\hline & $1 \mathrm{mo}$ & $167.7(9.5)$ & $170.3(5.9)$ & 0.3 & & \\
\hline & $6 \mathrm{mos}$ & $170.9(7.1)$ & $172.8(6.7)$ & 0.3 & & \\
\hline \multirow{3}{*}{$\begin{array}{l}\text { VILR }(\mathrm{BW} / \mathrm{s}) \\
\mathrm{n}=18\end{array}$} & $1 \mathrm{wk}$ & $65.1(13.5)$ & $67.1(14.0)$ & 0.1 & \multirow{3}{*}{$0.015^{*}$} & \multirow{3}{*}{0.247} \\
\hline & $1 \mathrm{mo}$ & $65.3(15.8)$ & $69.9(20.0)$ & 0.3 & & \\
\hline & $6 \mathrm{mos}$ & $62.6(13.9)$ & $64.6(17.3)$ & 0.1 & & \\
\hline \multirow{3}{*}{$\begin{array}{l}\operatorname{VALR}(\mathrm{BW} / \mathrm{s}) \\
\mathrm{n}=17\end{array}$} & $1 \mathrm{wk}$ & $48.1(8.1)$ & $49.8(9.1)$ & 0.2 & \multirow{3}{*}{$0.026^{*}$} & \multirow{3}{*}{0.101} \\
\hline & $1 \mathrm{mo}$ & $49.5(12.1)$ & $53.7(17.0)$ & 0.3 & & \\
\hline & $6 \mathrm{mos}$ & $47.6(9.3)$ & $49.1(12.5)$ & 0.1 & & \\
\hline \multicolumn{7}{|l|}{ FFS GROUP } \\
\hline \multirow{3}{*}{$\begin{array}{l}\text { Foot Angle (deg) } \\
n=15\end{array}$} & $1 \mathrm{wk}$ & $-3.0(4.3)$ & $-3.4(4.5)$ & 0.1 & \multirow{3}{*}{0.458} & \multirow{3}{*}{0.354} \\
\hline & $1 \mathrm{mo}$ & $-3.5(4.6)$ & $-3.7(5.1)$ & 0.04 & & \\
\hline & $6 \mathrm{mos}$ & $-2.0(4.6)$ & $-2.0(4.9)$ & 0.0 & & \\
\hline \multirow{3}{*}{$\begin{array}{l}\text { VILR (BW/s) } \\
n=13\end{array}$} & $1 \mathrm{wk}$ & $42.4(11.4)$ & $42.5(11.3)$ & 0.01 & \multirow{3}{*}{0.726} & \multirow{3}{*}{0.599} \\
\hline & $1 \mathrm{mo}$ & $43.9(15.2)$ & $42.5(11.8)$ & 0.1 & & \\
\hline & $6 \mathrm{mos}$ & $43.6(19.3)$ & $43.8(18.1)$ & 0.01 & & \\
\hline \multirow{3}{*}{$\begin{array}{l}\operatorname{VALR}(\mathrm{BW} / \mathrm{s}) \\
\mathrm{n}=13\end{array}$} & $1 \mathrm{wk}$ & $29.7(6.8)$ & $29.4(5.2)$ & 0.05 & \multirow{3}{*}{0.616} & \multirow{3}{*}{0.968} \\
\hline & $1 \mathrm{mo}$ & $29.1(6.2)$ & $28.8(5.2)$ & 0.05 & & \\
\hline & $6 \mathrm{mos}$ & $29.2(9.5)$ & $28.6(7.8)$ & 0.07 & & \\
\hline
\end{tabular}

P-values for condition and time-condition effects, *indicates significant change with distraction.

the average difference scores between undistracted and distracted conditions for cadence in the CAD group and foot angle in the FFS group (Table 3 ). We repeated this across the three post-retraining time points. We also calculated the standard error of the mean (SEM) values for cadence, foot angle, and loadrates in both the undistracted and distracted conditions for each time point (Table 3).

\section{Results}

Results of the 2-factor analysis for each group are presented in Table 2. There were no condition $x$ time interaction effects for variables of interest in the CAD group or in the FFS group, suggesting that no learning effect of the distraction task had occurred. There were no main effects for condition on cadence or foot angle. That is, there were no differences in cadence in the CAD group and foot angle in the FFS group between undistracted and distracted running. Upon further analysis of the individual participant data, we found a wide range of responses in both cadence and foot angle with distraction (Figure 2 and Figure 3). Because variables changed in both directions, we calculated a mean difference score between undistracted and distracted running for cadence and foot angle (Table 3). The mean difference values for the CAD group ranged between 3.4-6.3 steps/min across sessions, while the mean difference values for the FFS group ranged between 0.9 and 1.1 degrees. Finally, there was a main effect of condition on vertical loadrates, but only in the CAD group. The CAD runners exhibited small, but significant increases in VILR $(p=0.015)$ and VALR $(p=$ 0.026 ) with distracted running, while the FFS group did not (VILR $p=0.726$, VALR $p=0.616)$ (Table 2).

\section{Discussion}

This is the first study to examine changes in running mechanics with distraction. Specifically, we sought to determine if runners who had recently received gait retraining significantly altered their mechanics when distracted. We expected that, with distraction, the CAD group would exhibit reduced cadence and the FFS group would have reduced plantarflexion at initial contact. It was also hypothesized that vertical loadrates would increase with distraction for all runners. Finally, we examined whether a repeated use of the distraction task resulted in a learning effect on performance of the task over time. It was hypothesized runners would not become better at performing the task, and that it would remain equally distracting over time.

\section{Cadence}

In contrast to our hypothesis, cadence was unchanged with distracted running. While no difference was found, cadence varied considerably with distraction, with it increasing in some participants and decreasing in others (Figure 2). Fluctuations about the baseline within the calculated SEM values of between 2-3 steps/min would 

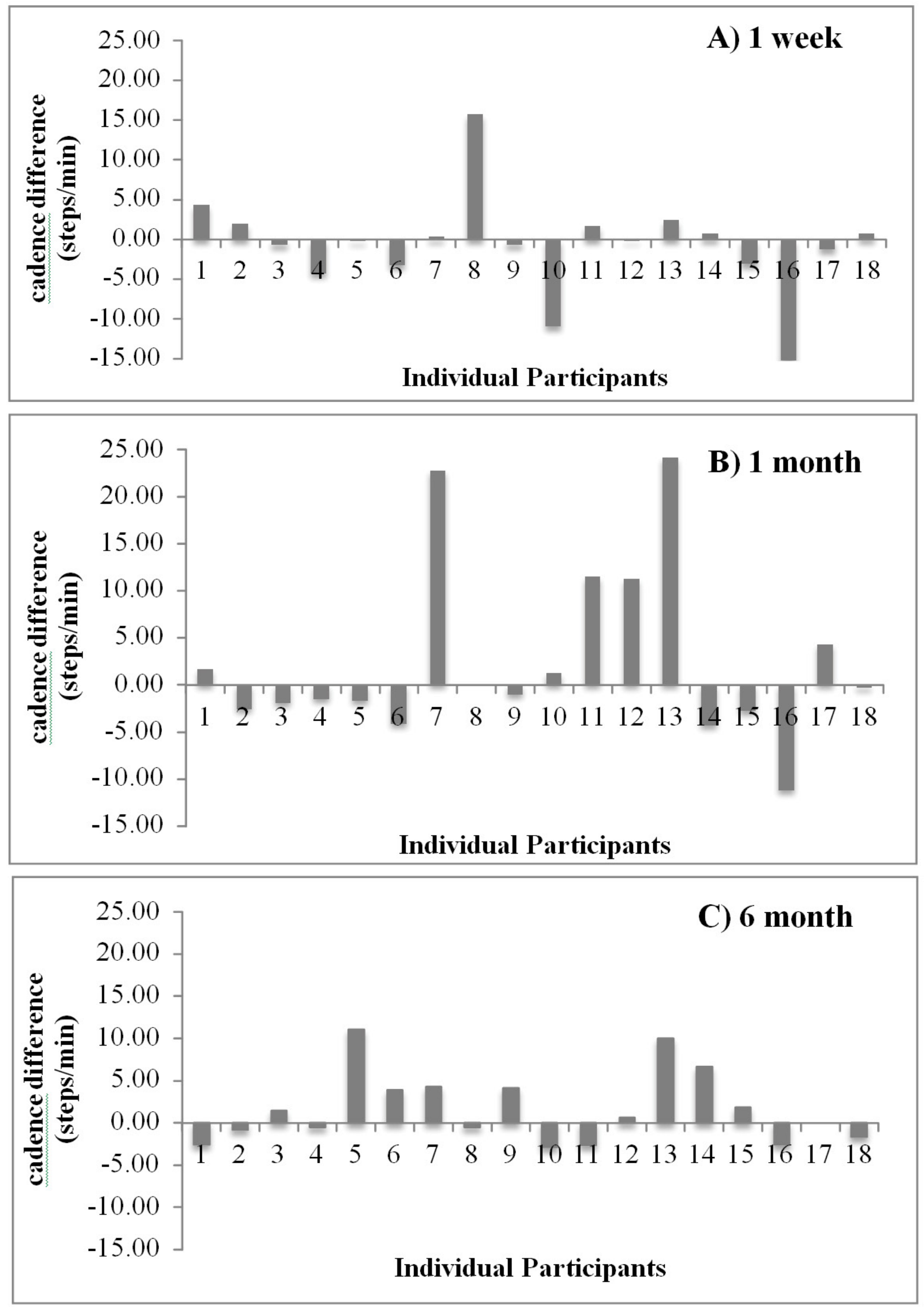

Figure 2: Individual difference scores of cadence in CAD group between undistracted and distracted running for A) 1 week, B) 1 month, C) 6 month post-intervention analyses. Positive value indicates an increase in cadence and negative indicates a decrease in cadence.

Note: participant 8 at 1 month and participant 17 at 6 months removed as outliers. 

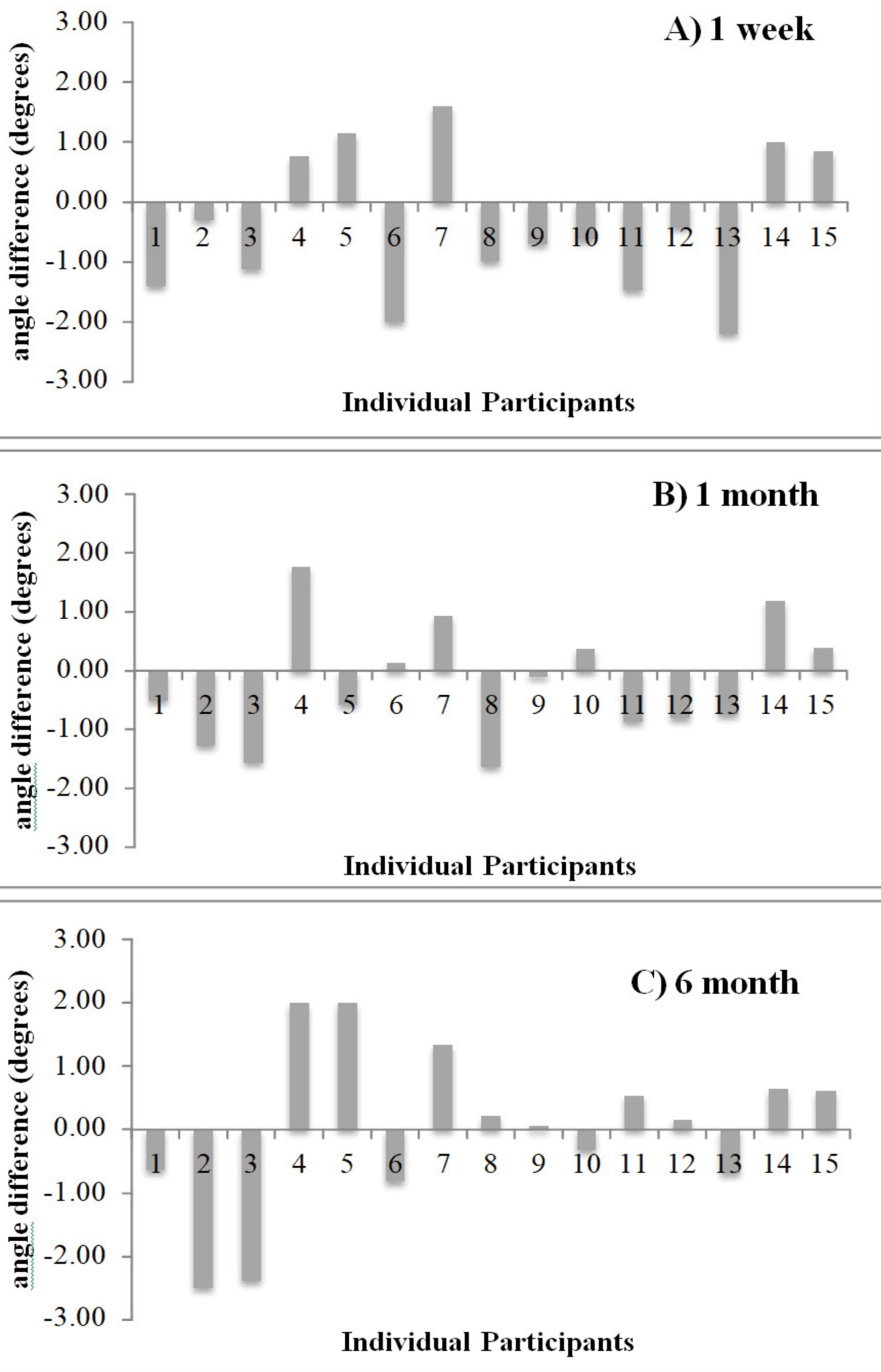

Figure 3: Individual difference scores of foot angle in FFS group between undistracted and distracted running for A) 1 week, B) 1 month, C) 6 month post-intervention analyses. (Negative values indicate more plantarflexed, and positive values indicate less plantarflexed at initial contact). 

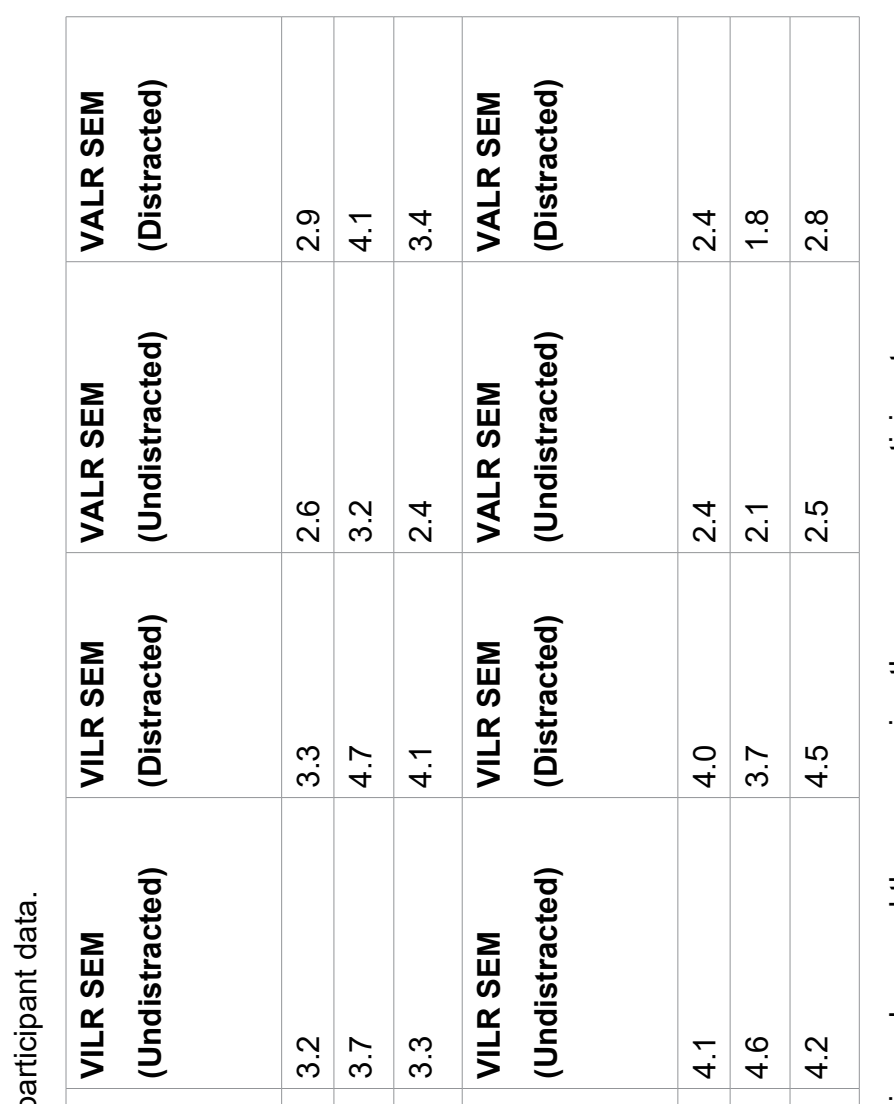

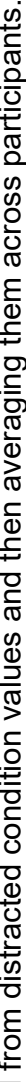

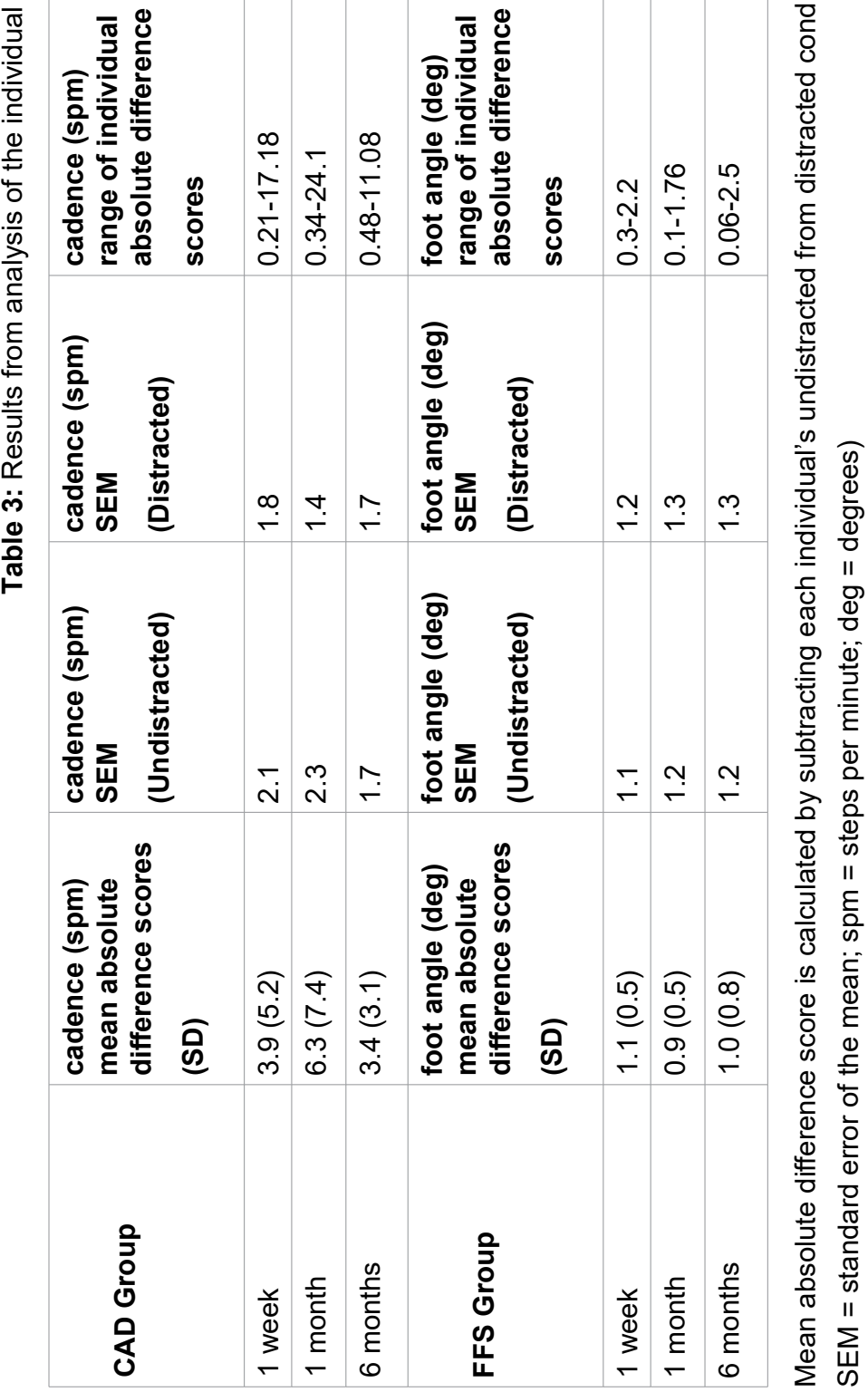

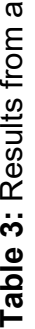
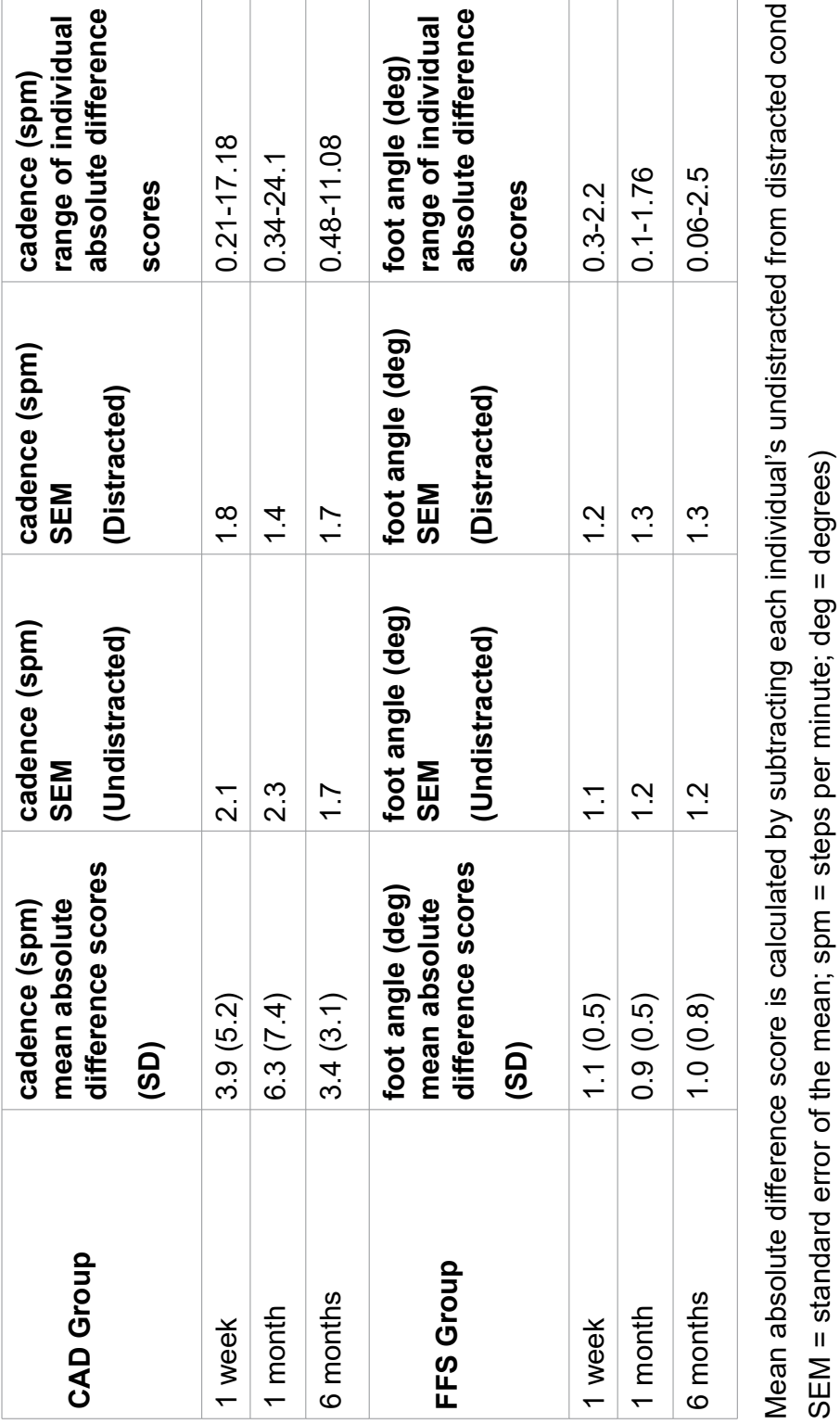
be expected (Table 3). However, there were numerous times when the cadence changed by 10 steps/min (over $3 \times$ SEM). If fact, there were some cases where increases and decreases of more than 20 steps/min (nearly 10x SEM) were noted. Additionally, individual participants did not respond in the same way across the three time points. For example, some increased cadence with distraction at one time point, then decreased at another (note participants 7, 10-14, Figure 2). This suggests that this motor pattern may not have been very stable.

\section{Foot angle}

Like cadence, foot angle also did not change with distraction as hypothesized. Similar to the CAD group, some of these runners' foot angles became less plantarflexed, while others became more plantarflexed with distraction (Figure 3). However, the absolute value of the difference scores only ranged between 0.9 and 1.1 degrees, well within the SEMs that ranged between 1.2-1.3 (Table 3). The largest difference noted across all three time points was only 2.5 degrees. Patterns appear to be more consistent for individual participants in the FFS group across the three time points than noted in the CAD group. These factors suggest that the motor pattern for the FFS group may be more stable than that for the CAD group.

\section{Vertical loadrates}

Retraining of cadence and footstrike pattern are typically aimed at reducing vertical loadrates [14,15], as excessive loadrates have been related to running injuries [7-9]. As expected, we did find significant overall increases in loadrates in the CAD group between undistracted and distracted running. This increase in loadrates in the absence of a change in cadence further supports the lack of relationship between these two variables as reported by Futrell, et al. [16]. However, it should also be noted that increases in vertical loadrates within the CAD group were small (1.7-4.6 BW/s) with small effect sizes $(d \leq 0.3)$ for each of the three follow-up time points (Table 2). These values were approximately within the SEM values of 2.1-4.7 BW/s. In contrast to the CAD group, loadrates did not change in the FFS group under the distracted condition, again suggesting this group may have been less affected by distraction than the CAD group.

\section{Interaction of condition $\times$ time}

As anticipated, the lack of significant interaction effect of condition $x$ time, indicated that the differences between the undistracted and distracted conditions did not change over time. These results indicate the runners did not become better or worse at performing the distraction task with repeated use. We believe the brief nature of the test ( 20 seconds) as well as the time between tests reduced the chance for learning. This suggests that the Stroop Test could be used for studies involving repeated measures.
In summary, individual changes in retrained gait patterns were seen with distracted running. They appear to vary in both directions from the undistracted running; however retrained cadence appeared to be much more affected than retrained footstrike angle. Additionally, only the retrained cadence group demonstrated an increase in vertical loadrates with distraction. Further studies are warranted to determine how distraction influences other gait variables. While it is assumed that the distraction test helps to reveal the true habitual gait pattern, it is also possible that too much distraction can unnaturally alter gait. We modified the Stroop Test to reduce its cognitive demand, however comparison to other distraction tests is warranted.

\section{Funding}

This work was supported by student scholarship funds from the AMTI Force and Motion Foundation. In addition, half of the footwear provided to participants was donated by Inov-8LLC. Equipment was loaned by Noraxon, USA.

\section{Conflicts of Interest}

We have no financial interests or conflicts of interest to disclose.

\section{References}

1. Fong DTP, Chan YY (2010) The use of wearable inertial motion sensors in human lower limb biomechanics studies: A systematic review. Sensors 10: 11556-11565.

2. McCambridge J, Witton J, Elbourne DR (2014) Systematic review of the Hawthorne effect: New concepts are needed to study research participation effects. J Clin Epidemiol 67: 267-277.

3. Paradis E, Sutkin G (2017) Beyond a good story: from Hawthorne Effect to reactivity in health professions education research. Med Educ 51: 31-39.

4. Malchow C, Fiedler G (2016) Effect of observation on lower limb prosthesis gait biomechanics: Preliminary results. Prosthet Orthot Int 40: 739-743.

5. Dai B, Cook RF, Meyer EA, et al. (2017) The effect of a secondary cognitive task on landing mechanics and jump performance. Sports Biomech 0: 1-14.

6. Weerdesteyn V, Schillings AM, van Galen GP, Duysens $J(2003)$ Distraction affects the performance of obstacle avoidance during walking. J Mot Behav 35: 53-63.

7. Messier SP, Davis shala E, Curl WW, Lowery RB, Pack RJ (1991) Etiologic factors associated with patellofemoral pain in runners. Med Sci Sports Exerc 23: 1008-1015.

8. Milner CE, Ferber R, Pollard CD, Hamill J, Davis IS (2006) Biomechanical factors associated with tibial stress fracture in female runners. Med Sci Sports Exerc 38: 323-328.

9. Pohl MB, Hamill J, Davis IS (2009) Biomechanical and anatomic factors associated with a history of plantar fasciitis in female runners. Clin J Sport Med 19: 372-376.

10. Futrell EE, Gross KD, Reisman D, Mullineaux DR, Davis IS (2020) Transition to forefoot strike reduces load rates more effectively than altered cadence. J Sport Health Sci 9: 248257. 
11. Stroop JR (1935) Studies of interference in serial verbal reactions. J Exp Psychol 18: 643- 662.

12. Leys C, Ley C, Klein O, Bernard P, Licata L (2013) Detecting outliers: Do not use standard deviation around the mean, use absolute deviation around the median. J Exp Soc Psychol 49: 764-766.

13. Mullineaux DR, Irwin G (2017) Error and anomaly detection for intra-participant time-series data. Int Biomech 4: 28-35.

14. Willson JD, Ratcliff OM, Meardon SA, Willy RW (2015) Influence of step length and landing pattern on patellofemoral joint kinetics during running. Scand J Med Sci Sports 25: 736-743.
15. Willy RW, Buchenic L, Rogacki K, Ackerman J, Schmidt A, et al. (2016) In-field gait retraining and mobile monitoring to address running biomechanics associated with tibial stress fracture. Scand J Med Sci Sports 26: 197-205.

16. Futrell EE, Jamison ST, Tenforde AS, Davis IS (2018) Relationships between habitual cadence, footstrike, and vertical load rates in runners. Med Sci Sports Exerc 50: 1837-1841. 